\title{
Avaliação de três madeiras nativas do cerrado goiano visando à utilização energética
}

\author{
Humberto Fauller de SIQUEIRAㄹ, Evelym Poliana Santos PATRÍCIO², Michael Douglas Roque LIMA³, \\ José Benedito GUIMARÃES JUNIOR², Angélica de Cássia Oliveira CARNEIRO¹, \\ Paulo Fernando TRUGILHO ${ }^{3}$, Thiago de Paula PROTÁSIO²* \\ ${ }_{1}^{1}$ Programa de Pós-Graduação em Ciência Florestal, Universidade Federal de Viçosa, Viçosa, MG, Brasil. \\ (Orcid: 0000-0001-5176-8448; 0000-0002-5992-3059) \\ ${ }^{2}$ Universidade Federal Rural da Amazônia, Parauapebas, PA, Brasil. (Orcid: 0000-0002-2177-3825; *) \\ 3 Programa de Pós-Graduação em Ciência e Tecnologia da Madeira, Universidade Federal de Lavras, Lavras, MG, Brasil. \\ (Orcid: 0000-0002-4789-1205; 0000-0002-9066-1069; 0000-0002-6230-5462) \\ *E-mail: depaulaprotasio@gmail.com (Orcid: 0000-0002-5560-8350)
}

Recebido em 11/05/2020; Aceito em 15/09/2020; Publicado em 27/09/2020.

\begin{abstract}
RESUMO: Estudos que visem ampliar o conhecimento das madeiras oriundas do cerrado podem contribuir com o correto manejo das espécies. O objetivo deste estudo foi avaliar a qualidade da madeira e do carvão vegetal de três espécies do cerrado (Tachigali vulgaris, Myracrodruon urundeuva e Amburana cearensis) para fins energéticos. Foram amostradas duas árvores por espécie e, destas, foram retirados discos da base, a 25, 50, 75 e $100 \%$ da altura total. Para a madeira, determinou-se longitudinalmente a densidade básica, composição química, estoque de carbono, poderes caloríficos superior (PCS) e inferior (PCI) e densidade energética. As carbonizações da madeira foram realizadas em forno elétrico, com temperatura final de $450{ }^{\circ} \mathrm{C}$ e taxa de aquecimento de $1,67^{\circ} \mathrm{C} \cdot \mathrm{min}^{-1}$. A posição longitudinal de amostragem não influenciou a densidade da madeira. A madeira de $M$. urundeuva apresentou maior densidade básica e PCS, bem como menor teor de materiais voláteis. O carvão da espécie T. vulgaris apresentou a menor densidade aparente $\left(298 \mathrm{~kg} \cdot \mathrm{m}^{-3}\right)$ e baixo teor de cinzas (1,14\%), porém elevado teor de carbono fixo e PCS. O carvão vegetal da espécie $M$. urundeuva se destacou com elevada densidade relativa aparente $\left(475 \mathrm{~kg} \cdot \mathrm{m}^{-3}\right)$. A ordem das melhores espécies é: $M$. urundeuva, A. cearensis e T. vulgaris.
\end{abstract}

Palavras-chave: energia renovável; espécies nativas; potencial energético; qualidade do carvão.

\section{Assessment of three native woods from cerrado of Goiás for the energy purposes}

\begin{abstract}
Studies aimed at expanding the knowledge of woods from cerrado can contribute to the correct management of the species. The aim of this study was to evaluate the quality of wood and charcoal of three species from cerrado (Tachigali vulgaris, Myracrodruon urundeuva, and Amburana cearensis) for energy purposes. Two trees per species were sampled and, from these, disks were removed from the base, at 25, 50, 75, and $100 \%$ of the total height. For wood, the properties basic density, chemical analysis, carbon stock, higher (HHV) and lower (LHV) heating values were determined longitudinally. Carbonizations were carried out in a muffle furnace with a final temperature of $450^{\circ} \mathrm{C}$ and heating rate of $1.67^{\circ} \mathrm{C} \cdot \mathrm{min}^{-1}$. There was no effect of longitudinal position under the wood basic density. Myracrodruon urundeuva wood had the highest basic density and HHV, as well as the lowest content of volatile matters. The Tachigali vulgaris charcoal had the lowest bulk density $\left(298 \mathrm{~kg} \cdot \mathrm{m}^{-3}\right)$ and low ash content $(1.14 \%)$, but a high content of fixed carbon and HHV. Charcoal of the species $M$. urundeuva stood out with high apparent relative density $\left(475 \mathrm{~kg} \cdot \mathrm{m}^{-3}\right)$. The order of the best species is $M$. urundeuva, $A$. cearensis, and T. vulgaris.
\end{abstract}

Keywords: renewable energy; native species; energy potential; charcoal quality.

\section{INTRODUÇÃO}

O cerrado brasileiro é o único bioma sul-americano que possui zonas de contatos biogeográficos distintas, devido limitar-se ao norte com o bioma Amazônia; a leste e nordeste, com a Caatinga; a sudoeste, com o Pantanal; e a sudeste, com a Mata Atlântica (SILVA; SANTOS, 2005). Estima-se que o cerrado ocupe uma área de $2.036 .448 \mathrm{~km}^{2}$ no Brasil, correspondendo a $22 \%$ do território nacional, segundo dados do Ministério do Meio Ambiente (MMA, 2020).

No entanto, a exploração e uso predatório das espécies florestais do bioma cerrado culminam na escassez de matérias-primas potenciais para o setor energético e madeireiro. Isso ocorre, principalmente, em virtude da falta de conhecimento tecnológico e anatômico de grande parte dessas espécies, evidenciando a necessidade de pesquisas científicas relacionadas à qualidade da madeira de espécies provenientes desse bioma. Com essas informações, é possível contribuir com a sustentabilidade do manejo florestal praticado no cerrado, com o Plano de Ação para Prevenção e Controle do Desmatamento e das Queimadas no Cerrado (PPCerrado), o qual, tem como um dos seus eixos temáticos o fomento ao manejo florestal de espécies nativas visando 
ampliar o plantio de florestas para fins energéticos e, finalmente, a conservação do bioma.

Madeiras com potencial adequado ao uso energético devem ter elevados valores de densidade básica, poder calorífico e lignina, além de baixo teor de cinzas. Essas propriedades culminarão no aumento do rendimento em carvão vegetal, redução de custo de produção e melhoria das propriedades desse insumo siderúrgico (PROTÁSIO et al., 2012; COSTA et al., 2014; PROTÁSIO et al., 2019). Para isso, é preciso diversificar as espécies e os usos, o que contribuirá para a redução da pressão sobre as espécies intensamente exploradas e que estão em risco de extinção.

Estudos evidenciam a qualidade energética da madeira e do carvão vegetal de espécies do cerrado no Brasil (VALE et al., 2010; TERRA, 2017; MARQUES et al., 2020). Costa et al. (2014) avaliando o potencial energético de cinco espécies nativas do cerrado mineiro (Luehea divaricata, Casearia sylvestris, Guazuma ulmifolia, Rapanea ferruginea e Trema micranta) reportaram variações importantes para a densidade básica $\left(0,364-0,625\right.$ g.cm $\left.{ }^{-3}\right)$, extrativos $(5,26-7,76 \%)$, lignina $(19,88-26,87 \%)$ e cinzas $(1,53-1,93 \%)$. Os mesmos autores destacaram rendimentos gravimétricos (30,88 $34,39 \%$ ) e propriedades promissoras do carvão vegetal dessas espécies, dentre elas a densidade relativa aparente $\left(0,255-0,475 \mathrm{~g} \cdot \mathrm{cm}^{-3}\right)$, poder calorífico superior $(7.135-$ $\left.7.730 \mathrm{kcal} . \mathrm{kg}^{-1}\right)$, carbono fixo $(77,2-81.0 \%)$ e rendimento em carbono fixo $(24,53-26,56 \%)$.

Diante disso, o objetivo deste trabalho foi avaliar a qualidade da madeira e do carvão vegetal de três espécies nativas do cerrado goiano (Myracrodruon urundeuva M. Allemão, Amburana cearensis (Allemão) A.C. Sm. e Tachigali vulgaris L.G. Silva \& H.C. Lima) visando à geração de energia.

\section{MATERIAL E MÉTODOS}

\subsection{Obtenção do material e análises da madeira}

Neste trabalho foram amostradas e avaliadas três espécies florestais de ocorrência no cerrado, são elas: aroeira-dosertão (Myracrodruon urundeuva M. Allemão), cerejeira (Amburana cearensis (Allemão) A.C. Sm.) e tachi-branco (Tachigali vulgaris L.G. Silva \& H.C. Lima). O material coletado foi proveniente de uma área de Cerrado localizada no município de Jataí, no Sudoeste do Estado de Goiás, Brasil.

Foram amostradas duas árvores por espécie e retirados discos de, aproximadamente, $10 \mathrm{~cm}$ de espessura da base, a $25,50,75$ e $100 \%$ da altura total dos indivíduos. Os discos foram seccionados em quatro cunhas, das quais duas opostas foram utilizadas na determinação da densidade básica, conforme recomendações da NBR 11941 (ABNT, 2003). As madeiras foram classificadas em classes de densidade (muito baixa: 200 - 300; baixa: 300 - 500; média: 500 - 750; alta: 750 - 1.000; e muito alta > $1.000 \mathrm{~kg} \cdot \mathrm{m}^{-3}$ ), de acordo com Csanády et al. (2015).

Para a realização das análises químicas, foram retiradas amostras (maravalhas) das cunhas restantes, por meio de uma plaina elétrica, de cada posição longitudinal amostrada. As análises físicas, químicas e energéticas da madeira foram realizadas por posição longitudinal de amostragem. O efeito da amostragem nas propriedades da madeira poderá subsidiar novos estudos sobre métodos laboratoriais e de campo para determinação da qualidade da madeira. Além disso, a definição precisa das variações das propriedades do lenho poderá ser relevante para a melhor gestão de equipamentos de conversão termoquímica.

Para quantificação dos teores de carbono (C) e hidrogênio $(\mathrm{H})$, em relação à massa seca da madeira, utilizouse um analisador universal da marca Elementar, modelo Vario Micro Cube. Posteriormente, a relação $\mathrm{H} / \mathrm{C}$ da madeira foi calculada. $\mathrm{O}$ estoque de carbono na madeira por unidade de volume (ECM) foi calculado por meio da Equação 1.

$$
\mathrm{ECM}=\mathrm{DB} *(\mathrm{C} / 100)
$$

em que: ECM é o estoque de carbono na madeira $\left(\mathrm{kg} \cdot \mathrm{m}^{-3}\right)$; DB é a densidade básica $\left(\mathrm{kg} \cdot \mathrm{m}^{-3}\right)$, e C é o carbono elementar (\%, base seca de madeira).

A composição química imediata da madeira foi realizada visando à determinação dos teores de materiais voláteis (TMV), cinzas (TCz) e, por diferença, o teor carbono fixo (TCF), conforme procedimento estabelecido na norma D1762-84 (ASTM, 2007).

O poder calorífico superior (PCS), base massa seca, foi determinado em calorímetro digital, marca IKA ${ }^{\circledR}$ C-200, conforme a norma E711-87 (ASTM, 2004). Enquanto que o poder calorífico inferior (PCI), base massa seca, foi estimado conforme a Equação 2 (BRAND, 2010).

$$
\mathrm{PCI}=\mathrm{PCS}-(600 * 9 \mathrm{H} / 100)
$$

em que: PCI é o poder calorífico inferior ( $\left.\mathrm{kcal}^{\mathrm{kg}} \mathrm{kg}^{-1}\right)$; PCS é o poder calorífico superior $\left(\mathrm{kcal}^{\mathrm{kg}} \mathrm{kg}^{-1}\right)$; e H é o teor de hidrogênio $(\%$, base seca de madeira).

As densidades energéticas superior ( $\mathrm{DE}_{\mathrm{PCS}}$ ) e inferior (DEPCI) foram obtidas por meio do produto da densidade básica pelos poderes caloríficos superior (PCS) e inferior (PCI), respectivamente.

\subsection{Carbonização e caracterização do carvão vegetal}

Foram carbonizadas, aproximadamente, 500 gramas de madeira por árvore amostrada, sendo utilizado em cada ensaio 10 cunhas (2 árvores x 5 posições longitudinais) provenientes dos discos retirados das árvores. As amostras de madeira foram previamente secas em estufa a $103 \pm 2^{\circ} \mathrm{C}$ até a massa constante.

As carbonizações foram realizadas em forno elétrico, tipo mufla, tendo como temperatura inicial $100^{\circ} \mathrm{C}$ e temperatura final $450^{\circ} \mathrm{C}$, considerando-se uma taxa de aquecimento de $1,67^{\circ} \mathrm{C} \mathrm{min}^{-1}\left(100^{\circ} \mathrm{C} \mathrm{h} \mathrm{h}^{-1}\right)$. O tempo total de carbonização foi de 4 horas e 30 minutos e a temperatura máxima foi mantida por 30 minutos, conforme realizado por Costa et al. (2014) e Protásio et al. (2021).

O rendimento gravimétrico em carvão vegetal (RGCV) foi obtido por meio da divisão da massa seca de carvão vegetal pela massa seca de madeira. $O$ rendimento em carbono fixo (RCF) foi obtido mediante o produto do rendimento gravimétrico em carvão vegetal (RGC) e o teor de carbono fixo do carvão vegetal.

Para as determinações da densidade relativa aparente, composição química imediata, poder calorífico superior e estoque de carbono fixo para o carvão vegetal, seguiram-se os mesmos procedimentos utilizados para madeira (ver tópico 2.1). 


\subsection{Análise Estatística}

$\mathrm{Na}$ avaliação das características da madeira utilizou-se um delineamento inteiramente casualizado (DIC) disposto em esquema fatorial 3 x 5, ou seja, considerando como níveis dos fatores as três espécies e as cinco posições longitudinais de amostragem no tronco das árvores e duas repetições (duas árvores). Para a avaliação dos rendimentos da carbonização e características do carvão vegetal utilizou-se um DIC com três tratamentos (três espécies) e dez repetições (2 árvores x 5 posições longitudinais). Para análise dos dados das carbonizações, o efeito de posição longitudinal não foi considerado, uma vez que ensaios preliminares demonstraram que este fator não influenciou a densidade da madeira (principal característica física que influencia a aplicação do carvão vegetal como biorredutor). Dessa forma, foi possível aumentar o número de graus de liberdade do erro nas análises de variância para verificar o efeito de espécie nas propriedades do carvão vegetal.

Para validação das análises de variância foram testadas as hipóteses básicas de independência, homogeneidade de variâncias e normalidade dos resíduos do modelo estatístico utilizado. Quando verificado efeito significativo de espécie, foi utilizado o teste de Scott-Knott em nível de 5\% de significância. Já para avaliação do efeito da posição longitudinal foram ajustados modelos de regressão linear simples.
Todas as análises estatísticas foram realizadas utilizandose o software SISVAR (FERREIRA et al., 2019).

\section{RESULTADOS}

\subsection{Propriedades químicas, físicas e energéticas da madeira}

Não houve efeito da posição longitudinal e da interação (Espécie x Posição) sob a densidade básica (DB), densidade energética (DE), estoque de carbono (EC), teor de hidrogênio $(\mathrm{H})$, teor de carbono (C) e relação hidrogênio/carbono (H/C). Foi verificado efeito significativo de espécie sob as propriedades DB, EC, H e H/C (Tabelas 1 e 2). Os baixos coeficientes de variação encontrados confirmam a precisão do experimento e a adequação do delineamento estatístico utilizado para análise da qualidade da madeira.

Na Figura 1, observam-se os valores médios de DB e EC das espécies avaliadas. De acordo com as classes de densidade básica propostas por Csanády et al. (2015), a M. urundeuva (706 kg.m ${ }^{-3}$ ) e $A$. cearensis (663 kg.m ${ }^{-3}$ ) são madeiras de média densidade, enquanto que o T. vulgaris (493 kg. $\left.\mathrm{m}^{-3}\right)$ possui baixa densidade. O EC seguiu a mesma tendência da DB, sendo o maior valor médio verificado para $M$. urundeuva $(341$ kg. $\mathrm{m}^{-3}$ ) e menor para T. vulgaris $\left(236 \mathrm{~kg} \cdot \mathrm{m}^{-3}\right)$.

Tabela 1. Resumo das análises de variância da densidade básica, estoque de carbono e densidades energéticas da madeira de três espécies nativas do cerrado goiano.

Table 2. Summary of analyses of variance of the basic density, carbon stock, and energy density of three native species from the Cerrado of Goiás.

\begin{tabular}{lccccc}
\hline \multirow{2}{*}{ FV } & \multirow{2}{*}{ GL } & \multicolumn{4}{c}{ Quadrado Médio } \\
\cline { 3 - 6 } & & $\mathrm{DB}\left(\mathrm{kg} \cdot \mathrm{m}^{-3}\right)$ & $\mathrm{EC}\left(\mathrm{kg} \cdot \mathrm{m}^{-3}\right)$ & $\mathrm{DE}_{\mathrm{PCS}}\left(\mathrm{Gcal} \cdot \mathrm{m}^{-3}\right)$ & $\mathrm{DE}_{\mathrm{PCI}}\left(\mathrm{Gcal} \cdot \mathrm{m}^{-3}\right)$ \\
\hline Espécie (E) & 2 & $126722,70^{*}$ & $30238,590^{*}$ & $2,925^{*}$ & $2,507^{*}$ \\
Posição (P) & 4 & $3474,674^{\mathrm{ns}}$ & $1768,187^{\mathrm{ns}}$ & $0,116^{\mathrm{ns}}$ & $0,100^{\mathrm{ns}}$ \\
E x P & 8 & $2205,681^{\mathrm{ns}}$ & $651,873^{\mathrm{ns}}$ & $0,058^{\mathrm{ns}}$ & $0,049 \mathrm{~ns}$ \\
Erro & 15 & 1949,624 & 891,024 & 0,039 & 0,033 \\
\hline Média & & 620 & 298 & 2,994 & 2,783 \\
\hline CVe & 7,11 & 10,00 & 6,65 & 6,56 \\
\hline
\end{tabular}

FV: fator de variação; GL: grau de liberdade; CVe: coeficiente de variação experimental; DB: densidade básica; EC: estoque de carbono; DEPCs: densidade energética base poder calorífico superior; DEpCr: densidade energética base poder calorífico inferior; *: significativo a $5 \%$ pelo teste F; ns: não significativo a $5 \%$ pelo teste $\mathrm{F}$.

Tabela 2. Resumo das análises de variância da composição química elementar da madeira de três espécies nativas do cerrado goiano.

Table 2. Summary of analyses of variance of the wood ultimate analysis of three native species from the Cerrado of Goiás.

\begin{tabular}{lcccc}
\hline \multirow{2}{*}{ FV } & \multirow{2}{*}{ GL } & \multicolumn{3}{c}{ Quadrado Médio } \\
\cline { 3 - 5 } & & $\mathrm{H}(\%)$ & $\mathrm{C}(\%)$ & $\mathrm{H} / \mathrm{C}$ \\
\hline Espécie (E) & 2 & $0,447^{*}$ & $0,473^{\mathrm{ns}}$ & $0,038^{*}$ \\
Posição (P) & 4 & $0,031^{\mathrm{ns}}$ & $5,050^{\mathrm{ns}}$ & $0,001^{\mathrm{ns}}$ \\
E x P & 8 & $0,110^{\text {ns }}$ & $5,981^{\mathrm{ns}}$ & $0,002^{\text {ns }}$ \\
Erro & 15 & 0,086 & 3,278 & 0,001 \\
\hline Média & & 6,3 & 48,0 & 1,5 \\
\hline CVe $(\%)$ & & 4,70 & 3,77 & 1,63
\end{tabular}

FV: fator de variação; GL: grau de liberdade; CVe: coeficiente de variação experimental; $\mathrm{H}$ : teor de hidrogênio; C: teor de carbono; $\mathrm{H} / \mathrm{C}$ : relação hidrogênio/carbono. *: significativo a 5\% pelo teste $\mathrm{F}$; ns: não significativo a $5 \%$ pelo teste $\mathrm{F}$.

Dentre as espécies analisadas, a $A$. cearensis apresentou maior teor de $\mathrm{H}(6,48 \%)$ e maior relação $\mathrm{H} / \mathrm{C}(1,62)$. O teor de $C$ na madeira não diferiu significativamente entre as espécies avaliadas (Tabela 3).
O teor de materiais voláteis (TMV), teor de cinzas (TCZ), teor de carbono fixo (TCF), poder calorífico superior (PCS) e poder calorífico inferior (PCI) das madeiras estudadas apresentaram efeito significativo da interação espécie $\mathrm{x}$ posição longitudinal (Tabela 4), logo se procedeu ao desdobramento e avaliação do efeito da posição longitudinal dentro da espécie e o efeito da espécie dentro da posição longitudinal.

$\mathrm{Na}$ avaliação do teor de materiais voláteis (TMV), em função da posição longitudinal, observou-se um aumento gradual, com tendência de estabilização nas posições longitudinais próximas ao topo para as madeiras das espécies M. urundeuva e T. vulgaris, com médias de $80,51 \%$ e $82,60 \%$, respectivamente (Figura 2). A espécie $A$. cearensis apresentou valor médio de TMV superior às outras espécies $(83,78 \%)$. No entanto, foi observada baixa influência da posição longitudinal de amostragem no TMV da madeira de $A$. cearensis, sendo o coeficiente de determinação igual a 0,23. 


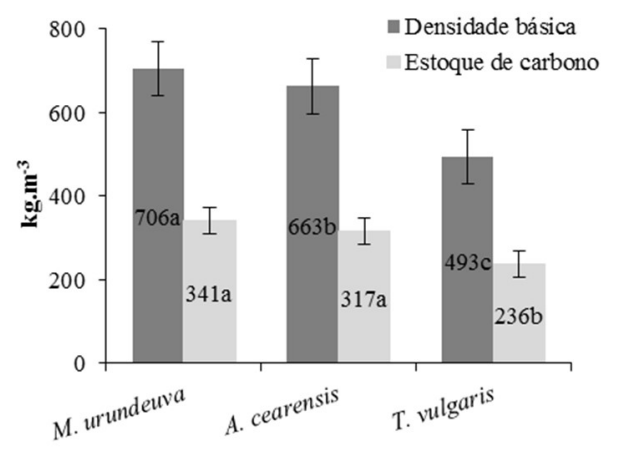

Figura 1. Valores médios da densidade básica e estoque de carbono da madeira de três espécies nativas do cerrado goiano. Diferentes letras na coluna representam diferença estatística de acordo com o teste de Scott-Knott $(p<0,05)$ e as barras de erro, o desvio padrão. Figure 2. Basic density and carbon stock of wood from three native species from the Cerrado of Goiás. Different letters in the column represent statistical difference according to the Scott-Knott test $(p$ $<0.05)$ and the error bars, the standard deviation.

Tabela 3. Valores médios da composição química elementar da madeira de três espécies nativas do cerrado goiano.

Table 3. Mean values of the wood ultimate analysis of three native species from the Cerrado of Goiás.

\begin{tabular}{lccc}
\hline Espécie & $\mathrm{H}(\%)$ & $\mathrm{C}(\%)$ & $\mathrm{H} / \mathrm{C}$ \\
\hline M. urundewva & $6,18 \mathrm{~b}$ & $48,29 \mathrm{a}$ & $1,51 \mathrm{~b}$ \\
A. cearensis & $6,48 \mathrm{a}$ & $47,92 \mathrm{a}$ & $1,62 \mathrm{a}$ \\
T. vulgaris & $6,08 \mathrm{~b}$ & $47,91 \mathrm{a}$ & $1,52 \mathrm{~b}$ \\
\hline Média & 6,25 & 48,04 & 1,55 \\
\hline CVe $(\%)$ & 4,70 & 3,77 & 1,63 \\
\hline
\end{tabular}

médios seguidos pela mesma letra na coluna não diferem entre si pelo teste de Scott-Knott $(p<0.05)$.

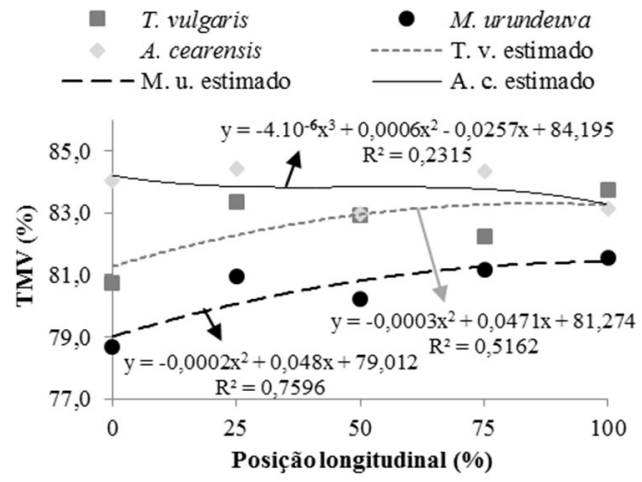

Figura 2. Teor de materiais voláteis (TMV) da madeira de três espécies nativas do cerrado em função da posição longitudinal. Figure 2. Content of volatile matter (VM) of the wood of three native species from the Cerrado as a function of longitudinal position.

Quanto ao teor de cinzas (TCz) na madeira, observa-se que a espécie $M$. urundeuva apresentou valor intermediário $(0,96 \%)$ entre as espécies $A$. cearensis $(1,10 \%)$ e $T$. vulgaris $(0,18 \%)$, com tendência de acréscimo gradual da base até a posição longitudinal central, com posterior decréscimo até o topo. Para espécie T. vulgaris há tendência de variação contrária, em que o $\mathrm{TCz}$ decresce da base até a posição central do fuste e, posteriormente, aumenta até o topo (Figura 3). Para a espécie $A$. cearensis, não foi confirmado efeito significativo da posição longitudinal da madeira sob o TCZ.

Tabela 4. Resumo das análises de variância para as características da madeira que apresentaram interação significativa entre espécie x posição longitudinal.

Table 4. Summary of analyses of variance for the characteristics in which there was a significant interaction between species $\mathrm{x}$ longitudinal position.

\begin{tabular}{lcccccc}
\hline \multirow{2}{*}{ FV } & \multirow{2}{*}{ GL } & \multicolumn{5}{c}{ Quadrado Médio } \\
\cline { 3 - 7 } & & TMV $(\%)$ & TCZ $(\%)$ & TCF $(\%)$ & PCS $\left(\mathrm{kcal}^{\circ} \mathrm{kg}^{-1}\right)$ & PCI $\left.\left(\mathrm{kcal}^{-}\right) \mathrm{kg}^{-1}\right)$ \\
\hline Espécie (E) & 2 & $27,37^{*}$ & $2,43^{*}$ & $29,48^{*}$ & $145001,07^{*}$ & $172989,92^{*}$ \\
Posição (P) & 4 & $3,11^{*}$ & $0,07^{*}$ & $2,25^{*}$ & $18448,87^{*}$ & $16960,53^{*}$ \\
Ex P & 8 & $1,59 *$ & $0,07^{*}$ & $2,02^{*}$ & $9788,73^{*}$ & $9502,66^{*}$ \\
Erro & 15 & 0,06 & 0,00 & 0,06 & 2492,30 & 3138,54 \\
\hline Média & & 82,30 & 0,74 & 16,96 & 4.827 & 4.489 \\
\hline CVe (\%) & & 0,30 & 2,10 & 1,43 & 1,03 & 1,25 \\
\hline
\end{tabular}

FV: fator de variação; GL: grau de liberdade; CVe: coeficiente de variação experimental; PCS: poder calorífico superior; PCI: poder calorífico inferior; TMV, TCZ e TCF: teores de materiais voláteis, cinzas e carbono fixo, respectivamente. *: significativo a $5 \%$ pelo teste F; ns: não significativo a $5 \%$ pelo teste F.

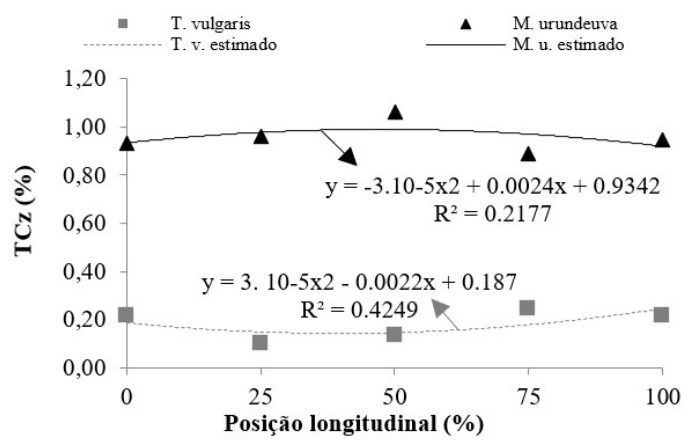

Figura 3. Teor de cinzas $(\mathrm{TCz})$ da madeira de três espécies nativas do cerrado em função da posição longitudinal.

Figure 3. Ash content (AC) of the wood of three native species from the Cerrado as a function of longitudinal position.
Com relação ao teor de carbono fixo (TCF), foi observado decréscimo no sentido base-topo para as espécies M. urundeuva e T. vulgaris (Figura 4). A espécie A. cearensis $(15,12 \%)$ não apresentou efeito significativo da posição longitudinal no TCF. A madeira das espécies $M$. urundeuva $(18,53 \%)$ e $T$. vulgaris $(17,22 \%)$ apresentaram os maiores valores de TCF.

Não houve efeito significativo da posição longitudinal no poder calorífico superior (PCS) da madeira das espécies $A$. cearensis (valor médio de $4.688 \mathrm{kcal}^{\mathrm{kg}} \mathrm{kg}^{-1}$ ) e $T$. vulgaris (valor médio de $=4.880 \mathrm{kcal}^{\mathrm{kg}} \mathrm{kg}^{-1}$ ). Por outro lado, a espécie $M$. urundeuva apresentou maior valor de PCS (4.911 kcal.kg-1) com tendência de decréscimo dessa propriedade no sentido longitudinal (Figura 5). 
Para A. cearensis, observou-se PCI médio de 4.338 kcal.kg1 e, o efeito da posição longitudinal não foi estatisticamente significativo. As espécies $M$. urundeuva (valor médio $=4.577$ kcal. $\mathrm{kg}^{-1}$ ) e $T$. vulgaris (valor médio $=4.553$ kcal. $\mathrm{kg}^{-1}$ ) apresentaram tendência de redução do PCI com o aumento da posição longitudinal (Figura 6).

Observa-se que os resultados encontrados para DEpcs e DEPCI, foram similares aos observados para a DB (ver Figura 1). A espécie $M$. urundewva apresentou os valores mais elevados para essa propriedade (Figura 7), sendo 3,47 Gcal.m 3 e 3,23 Gcal.m ${ }^{-3}$ para as densidades energéticas calculadas com base no PCS e PCI, respectivamente. A densidade energética da madeira da espécie $M$. urundeuva foi $44 \%$ maior comparativamente ao encontrado para a espécie $T$. vulgaris. Para a madeira de $A$. cearensis os valores da densidade energética foram intermediários comparativamente as demais espécies avaliadas.

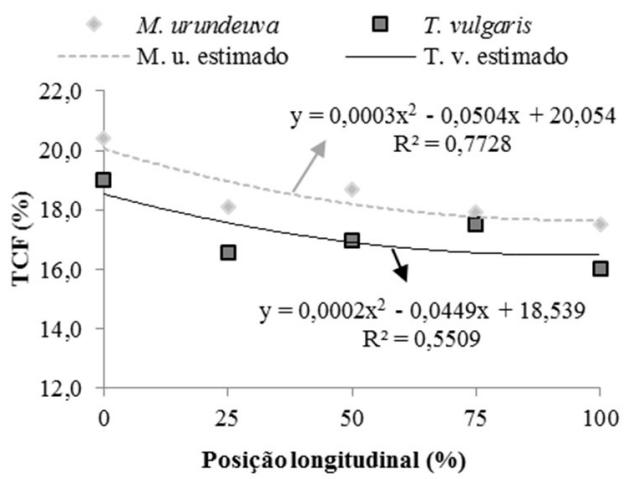

Figura 4. Teor de carbono fixo (TCF) da madeira de espécies nativas do cerrado em função da posição longitudinal.

Figure 4. Content of fixed carbon (FC) of the wood of native species from the Cerrado as a function of longitudinal position.

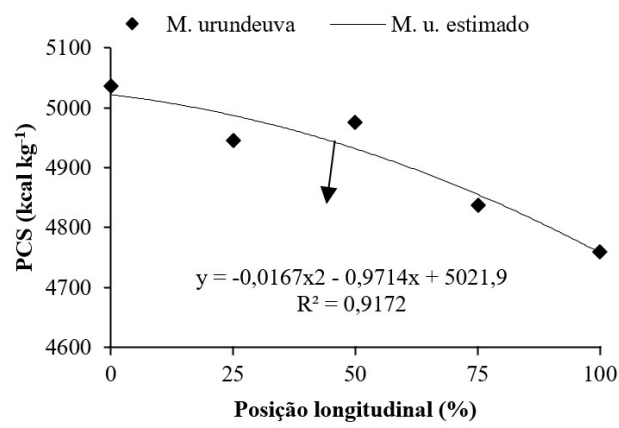

Figura 5. Relação entre o poder calorífico superior (PCS) da madeira de $M$. urundewva e a posição longitudinal.

Figure 5. Relationship between higher heating value (HHV) of the wood of M. urundewva and the longitudinal position.

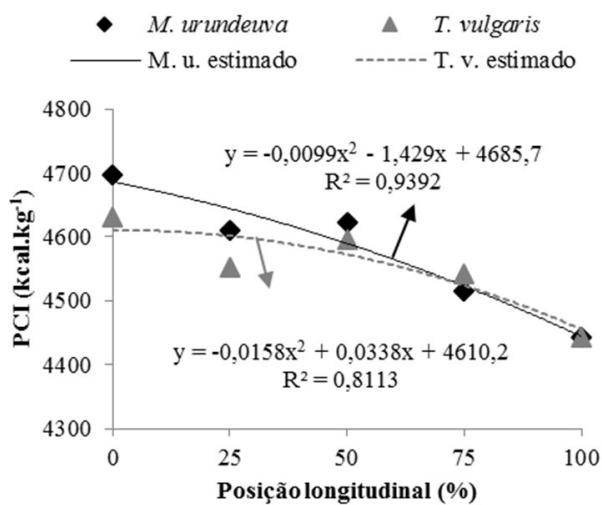

Figura 6. Relação entre o poder calorífico inferior (PCI) da madeira de espécies nativas do cerrado e a posição longitudinal de amostragem.

Figure 6. Relationship between lower heating value (LHV) of the wood of native species from the Cerrado and longitudinal position.

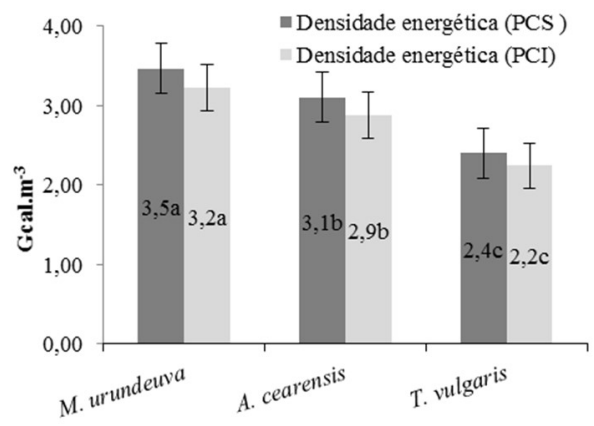

Figura 7. Valores médios das densidades energéticas da madeira de três espécies nativas do cerrado goiano. Diferentes letras na coluna representam diferença estatística de acordo com o teste de ScottKnott $(p<0,05)$ e as barras de erro, o desvio padrão.

Figure 7. Average values of energy density of wood from three native species from the Cerrado of Goiás. Different letters in the column represent statistical difference according to the Scott-Knott test $(p<0.05)$ and the error bars, the standard deviation.

\subsection{Rendimento e caracterização energética do carvão vegetal}

O RGCV apresentou valores médios de 35,34\%, 34,75\% e 32,67\% para as espécies M. urundeuva, Tachigali vulgaris e $A$. cearensis, respectivamente, não sendo detectado efeito significativo do fator espécie (Tabela 5). Resultado diferente foi verificado para o ECF e RCF. Portanto, pode-se afirmar que o efeito da espécie foi mais pronunciado para a qualidade do carvão vegetal e produtividade de carbono fixo.

Tabela 5. Resumo das análises de variância para rendimentos gravimétricos da carbonização e características do carvão vegetal.

Table 5. Summary of analyses of variance for gravimetric yields of the carbonization and characteristics of charcoal.

\begin{tabular}{lcccccc}
\hline \multirow{2}{*}{ Fator de variação } & Graus de & \multicolumn{5}{c}{ Quadrado Médio } \\
\cline { 3 - 7 } & liberdade & ECF $\left(\mathrm{kg} \cdot \mathrm{m}^{-3}\right)$ & RGCV $(\%)$ & RCF $(\%)$ & DRA $\left(\mathrm{kg} \cdot \mathrm{m}^{-3}\right)$ & PCS $\left(\mathrm{kcal} \cdot \mathrm{kg}^{-1}\right)$ \\
\hline Espécie & 2 & $33360,10^{*}$ & 19,72 ns & $22,92^{*}$ & $83519,33^{*}$ & $290156,70^{*}$ \\
Erro & 27 & 1435,41 & 10,24 & 5,23 & 2818,13 & 19277,59 \\
\hline Média & & 290,80 & 34,25 & 25,18 & 399,02 & 7615 \\
\hline CVe $(\%)$ & 13,03 & 9,34 & 9,11 & 13,30 & 1,82 \\
\hline
\end{tabular}

FV: fator de variação; GL: grau de liberdade; CVe: coeficiente de variação experimental; DRA: densidade relativa aparente; PCS: poder calorífico superior; TMV: teor de materiais voláteis; CZ: teor de cinzas; TCF: teor de carbono fixo. *: significativo a 5\% pelo Teste de F; ns: não significativo a $5 \%$ pelo Teste F. 
Houve efeito significativo da espécie nas propriedades físicas (DRA), energéticas (PCS) e químicas (TMV, TCZ e TCF) do carvão vegetal (Tabelas 5 e 6 ). Conforme esperado, para o teor de cinzas foi obtido elevado coeficiente de variação experimental indicando ampla variabilidade entre as amostras avaliadas. Quaisquer variações no teor de cinzas resultam em maior coeficiente de variação, pois essa propriedade apresenta menor proporção na composição química imediata do carvão vegetal (valor médio de $2,29 \pm 1,05 \%)$.

Os carvões vegetais das espécies $A$. cearensis e $M$. urundeuva tiveram os maiores valores de TMV e menores de TCF, além de elevados valores de TCz (Tabela 7).

As espécies $M$. urundeuva $\left(475 \mathrm{~kg} \cdot \mathrm{m}^{-3}\right)$ e $A$. cearensis $(425$ $\left.\mathrm{kg} . \mathrm{m}^{-3}\right)$ apresentaram maiores valores de DRA. A mesma tendência foi observada para o estoque de carbono fixo (Figura 8).

Tabela 6. Resumo das análises de variância para composição química imediata do carvão vegetal

Table 6. Summary of analyses of variance for the proximate composition of charcoal.

\begin{tabular}{lcccc}
\hline \multirow{2}{*}{ FV } & \multirow{2}{*}{ GL } & \multicolumn{3}{c}{ Quadrado Médio } \\
\cline { 3 - 5 } & & TMV (\%) & TCZ (\%) & TCF (\%) \\
\hline Espécie & 2 & $29,44^{*}$ & $11,34^{*}$ & $68,13^{*}$ \\
Erro & 27 & 2,11 & 0,35 & 1,91 \\
\hline Média & 24,39 & 2,29 & 73,32 \\
\hline CVe $(\%)$ & 5,95 & 26,02 & 1,89 \\
\hline
\end{tabular}

FV: fator de variação; GL: grau de liberdade; CVe: coeficiente de variação experimental; TMV: teor de materiais voláteis; TCZ: teor de cinzas; TCF teor de carbono fixo. *: significativo a $5 \%$ pelo Teste de $\mathrm{F}$; ns: não significativo a $5 \%$ pelo Teste $\mathrm{F}$.

Tabela 7. Valores médios de algumas características avaliadas no carvão vegetal

Table 7. Average values for some characteristics evaluated in charcoal.

\begin{tabular}{lccccc}
\hline Espécie & $\begin{array}{c}\text { TMV } \\
(\%)\end{array}$ & $\begin{array}{c}\text { TCF } \\
(\%)\end{array}$ & $\begin{array}{c}\text { TCz } \\
(\%)\end{array}$ & $\begin{array}{c}\text { PCS } \\
\left(\mathrm{kcal.kg}^{-1}\right)\end{array}$ & $\begin{array}{c}\text { RCF } \\
(\%)\end{array}$ \\
\hline M. urundeuva & $25,92 \mathrm{a}$ & $71,60 \mathrm{~b}$ & $2,48 \mathrm{~b}$ & $7419 \mathrm{~b}$ & $25,29 \mathrm{a}$ \\
A. cearensis & $24,72 \mathrm{a}$ & $72,04 \mathrm{~b}$ & $3,24 \mathrm{a}$ & $7697 \mathrm{a}$ & $23,53 \mathrm{~b}$ \\
T. vulgaris & $22,54 \mathrm{~b}$ & $76,32 \mathrm{a}$ & $1,14 \mathrm{c}$ & $7729 \mathrm{a}$ & $26,54 \mathrm{a}$ \\
\hline Média & 24,39 & 73,32 & 2,29 & 7615 & 25,12 \\
CVe $(\%)$ & 5,95 & 1,89 & 26,02 & 1,82 & 9,11 \\
\hline
\end{tabular}

TMV = teor de materiais voláteis (\%); TCF = teor de carbono fixo (\%); TCz: teor de cinzas (\%); PCS: poder calorífico superior $\left(\mathrm{kcal}^{\mathrm{kg}}{ }^{-1}\right)$; e RCF: rendimento em carbono fixo (\%). Valores médios seguidos pela mesma letra na coluna não diferem entre si pelo teste de Scott-Knott em nível de 5\% de significância.

\section{DISCUSSÃO}

\subsection{Propriedades químicas, físicas e energéticas da} madeira

A ausência de efeito estatístico da posição longitudinal e da interação dos fatores posição $\mathrm{x}$ espécie sob as propriedades da madeira (DB, DE, EC, H, C e H/C) indica similaridade das características no sentido base-topo do tronco (Tabela 1). Dessa forma, a amostragem da madeira destas espécies pode ser considerada como fração única para base de cálculos quantitativos. A ausência de variação significativa de DB nas distintas posições longitudinais indica que o carvão vegetal produzido com madeiras dessas espécies tende a apresentar propriedades físicas e mecânicas mais homogêneas. A DB é o principal índice utilizado para a avaliação de espécies para finalidade energética devido a sua relação com importantes parâmetros de qualidade do carvão vegetal, como densidade relativa aparente, densidade energética, resistência mecânica e produtividade dos fornos (DIAS et al., 2018; FORTALEZA et al., 2019).

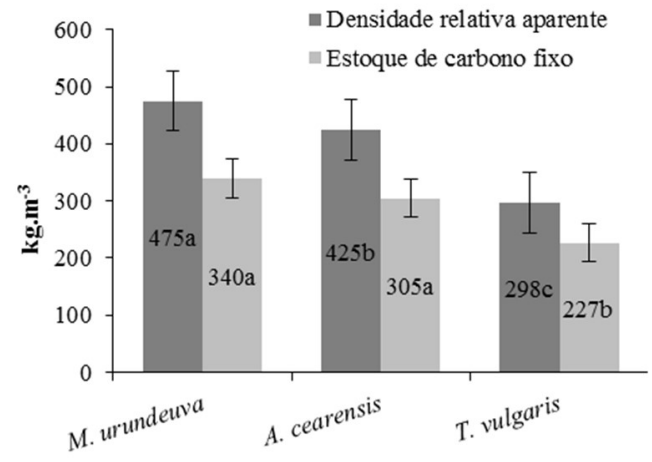

Figura 8. Densidade relativa aparente e estoque de carbono fixo do carvão vegetal de três espécies nativas do cerrado goiano. Diferentes letras na coluna representam diferença estatística de acordo com o teste de Scott-Knott $(p<0,05)$ e as barras de erro, o desvio padrão. Figure 8. Bulk density and fixed carbon stock of the charcoal from three native species from the Cerrado of Goiás. Different letters in the column represent statistical difference according to the ScottKnott test $(p<0.05)$ and the error bars, the standard deviation.

Vale et al. (2010) avaliando espécies do cerrado provenientes da região norte de Goiás, registraram DB média de $780 \mathrm{~kg} \cdot \mathrm{m}^{-3}$ para T. vulgaris, valor superior ao encontrado nessa pesquisa (Figura 1). Para a espécie M. urundeuva, Carvalho (2003) relatou que a madeira pode alcançar até $1.210 \mathrm{~kg} \cdot \mathrm{m}^{-3}$, enquanto que para $A$. cearensis, os resultados encontrados foram semelhantes aos relatados por Azevêdo et al. (2010), o qual obteve DB média de $630 \mathrm{~kg} \cdot \mathrm{m}^{-3}$. Madeiras com densidades elevadas culminam em carvões vegetais mais densos, resistentes e com maior densidade energética, portanto mais adequados ao uso siderúrgico (CARNEIRO et al., 2014). Para essa finalidade, as espécies $M$. urundeuva (706 $\mathrm{kg} . \mathrm{m}^{-3}$ ) e $A$. cearensis $\left(663 \mathrm{~kg} \cdot \mathrm{m}^{-3}\right.$ ) são recomendadas, pois apresentam valores de densidade acima de 500 kg.m ${ }^{-3}$ (SANTOS et al., 2011), o que reduzirá as preocupações associadas ao controle de umidade, bem como a quantidade de energia térmica liberada pela queima direta dessas madeiras será maximizada. Por outro lado, a madeira de $T$. vulgaris (493 kg.m ${ }^{-3}$ ) não apresenta densidade adequada para produção de carvão siderúrgico (PROTÁSIO et al., 2021). No entanto, o carvão vegetal dessa espécie pode ser destinado a finalidades domésticas, como cocção de alimentos ou churrasco.

Os valores médios verificados para os componentes elementares $(\mathrm{C}$ e $\mathrm{H})$ e a relação $\mathrm{H} / \mathrm{C}$ estão coerentes aos relatados na literatura para combustíveis lignocelulósicos (LIMA et al., 2011; SOARES et al., 2014). Madeiras com teores elevados de $\mathrm{H}$ e $\mathrm{C}$ liberam mais energia no processo de combustão, o que influencia positivamente o valor calórico do biocombustível (ADAMOVICS et al., 2018). Para a madeira de Eucalyptus, importante recurso energético utilizado no Brasil, tem sido relatado na literatura teores de $\mathrm{C}$ variando de 43,97 a 48,53\% e de $\mathrm{H}$ variando de 6,01 a 6,43\%. Dessa forma, a relação $\mathrm{H} / \mathrm{C}$ para madeira de Eucalyptus varia entre 1,59 a 1,64 (OLIVEIRA et al., 2010; NEVES et al., 
2011; REIS et al., 2012; SOARES et al., 2014; PROTÁSIO et al., 2019). Apesar de similaridades nos teores de $\mathrm{C}$ e $\mathrm{H}$ das madeiras destinadas ao uso energético, pequenas alterações nas proporções desses elementos podem influenciar a energia liberada na combustão desses materiais lignocelulósicos (PROTÁSIO et al., 2011). Nesta pesquisa não foram encontradas diferenças significativas no teor de $\mathrm{C}$ nas madeiras do cerrado analisadas. Contudo, os teores de $\mathrm{H}$ diferiram entre as espécies $M$. urundeuva, $A$. cearensis e $T$. vulgaris. Com base na relação $\mathrm{H} / \mathrm{C}$, a espécie $A$. cearensis obteve maior valor médio $(1,62)$ em virtude do elevado teor de H (6,48\%). Entretanto, a espécie apresentou menor PCS $\left(4.688{\left.\mathrm{kcal} . \mathrm{kg}^{-1}\right)}^{-1}\right.$, provavelmente devido ao seu maior TCZ $(1,10 \%)$.

De acordo com Chaves et al. (2013), as cinzas resíduos do processo de combustão da madeira e são indesejáveis para a produção de energia. Visto que, o acúmulo dessas cinzas danificam os equipamentos e aumentam a periodicidade de limpezas nos sistemas que utilizam a madeira como fonte energética. Logo, baixos teores de cinzas são requeridos para a utilização energética da madeira, especialmente considerando processos termoquímicos de conversão.

Os valores de TCz verificados na madeira das espécies $T$. vulgaris e $M$. urundewva, foram similares aos trabalhos relatados na literatura para madeira de eucalipto com valores médios entre 0,07\% e 1,00\% (PROTÁSIO et al., 2011; NEVES et al., 2011; SILVA et al., 2015; COSTA et al., 2017).

O valor médio de TCF obtido nesse estudo foi de 16,96\%, variando de 15,12\% (A. cearensis) a 18,53\% ( $M$. urundewva). Madeiras com valores médios de TCF elevados apresentam queima mais lenta, provocando o aumento do tempo de residência nos aparelhos de queima (MORAES et al., 2019). Por outro lado, madeiras com menor TCF e, consequentemente, maior TMV queimam mais rapidamente e apresentaram maior facilidade de ignição. O TCF e o TMV são frações químicas importantes na dinâmica de liberação de energia durante a queima da madeira, pois afetam a reação de oxidação, consequentemente, a taxa de queima e a homogeneidade da chama na câmara de combustão (SANTOS et al., 2013). A maior proporção de TMV permite a madeira entrar em ignição mais facilmente (LIMA et al., 2020). Contudo, destaca-se a necessidade de equilíbrio entre o TCF e TMV na madeira para o uso energético. Considerando as espécies analisadas, $A$. cearensis deve apresentar mais facilidade para entrar em ignição e maior reatividade durante a combustão devido o maior valor médio reportado de materiais voláteis $(83,78 \%)$.

O PCS é uma das principais variáveis consideradas na comparação e seleção de espécies para fins energéticos, uma vez que está relacionado com a quantidade de energia liberada pela madeira durante a sua combustão (CARNEIRO et al., 2014). Apesar da espécie $A$. cearensis (4.688 kcal.kg-1) apresentar menor PCS em relação às espécies $M$. urundeuva e T. vulgaris, este valor é maior que os dados normalmente reportados para madeiras de Eucalyptus. Os valores encontrados para esta propriedade foram maiores se comparados aos reportados por Lima et al. (2011) e Soares et al. (2014) avaliando madeiras de Eucalyptus para fins energéticos, com idade entre 5 e 6 anos, com valores médios variando de $4.547 \mathrm{kcal} \mathrm{kg}^{-1}$ a $4.681 \mathrm{kcal} . \mathrm{kg}^{-1}$, respectivamente.

O PCS médio da madeira das espécies do cerrado (4.827 kcal. $\left.\mathrm{kg}^{-1}\right)$ foi superior às madeiras convencionalmente utilizadas para a finalidade energética, o que credencia todas as espécies avaliadas nesse estudo com elevado potencial energético. Além disso, o teor médio de cinzas pode ser considerado baixo $(0,74 \%)$, ou seja, a cada tonelada de madeira destinada a combustão serão produzidos $7,4 \mathrm{~kg}$ de cinzas.

No presente estudo foi verificado que o PCS da madeira decresceu aproximadamente $1,75 \%$ de acordo com o aumento da posição longitudinal para a espécie $M$. urundeuva (Figura 5), essa variação pode ser atribuída aos teores de C e $H$ que variaram entre $50,7 \%-45,6 \%$ e $6,3 \%-5,9 \%$, respectivamente entre base e topo. Contudo essa variação não é significativa do ponto de vista prático, visto que, para produção de energia consideram-se diferenças significativas no PCS àquelas maiores que $300 \mathrm{kcal} \cdot \mathrm{kg}^{-1}$, conforme relatado por Brand (2010).

Outra propriedade de interesse na classificação de espécies para geração de calor é a densidade energética que considera a energia contida num determinado volume de madeira (LIMA et al., 2011; LOUREIRO et al., 2019). Protásio et al. (2019) em estudos com clones de Eucalyptus, com aproximadamente sete anos de idade, encontraram DEPCI de $2,31 \mathrm{Gcal}^{-3}$. As espécies $A$. cearensis e $M$. urundeuva apresentaram densidades energéticas $25 \%$ a $40 \%$ superiores comparativamente aos valores reportados na literatura para clones jovens de Eucalyptus. Essas diferenças podem ser atribuídas aos distintos valores de densidade básica das madeiras de $A$. cearensis e $M$. urundeuva (663 a $706 \mathrm{~kg} \cdot \mathrm{m}^{-3}$ ) e dos clones de Eucalyptus (em média: $534 \mathrm{~kg} \cdot \mathrm{m}^{-3}$ ), conforme reportado por Protásio et al. 2019. A maior densidade da madeira está diretamente relacionada ao acréscimo da densidade energética (LOUREIRO et al., 2019). Isto indica que as madeiras das espécies $A$. cearensis e $M$. urundeuva apresentam considerável potencial para a geração de energia térmica por permitir melhor uso dos equipamentos de conversão, como caldeiras. Além disso, os resultados desta pesquisa demonstram que o efeito da densidade da madeira é o principal fator responsável por ampliar as variações da densidade energética. A densidade básica das madeiras analisadas apresentou amplitude de variação de 43\% (493 a $706 \mathrm{~kg} \cdot \mathrm{m}^{-3}$ ), enquanto o PCS variou apenas 5\% (4911 a 4688 kcal.kg-1). Essas observações podem ser confirmadas analisando os resultados obtidos para o lenho de T. vulgaris que apresentou PCS de $4880 \mathrm{kcal} . \mathrm{kg}^{-1}$, ou seja, próximo ao observado para a espécie $M$. urundeuva (4911 kcal. $\left.\mathrm{kg}^{-1}\right)$. No entanto, a densidade básica da madeira de T. vulgaris foi de

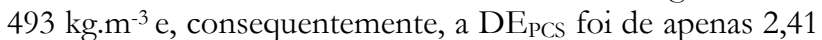
Gcal. $\mathrm{m}^{-3}$. Por outro lado, a madeira de M. urundenva com densidade básica consideravelmente superior, apresentou DEpcs de 3,47 Gcal.m $\mathrm{m}^{-3}$ ou 44\% maior comparativamente a espécie T. vulgaris. Marques et al. (2020) estudaram o potencial energético de madeiras do cerrado e constataram que a espécie Annona coriacea apresentou DB de $532 \mathrm{~kg} \cdot \mathrm{m}^{-3}$ e DEPCS 2,38 Gcal. $\mathrm{m}^{-3}$. Para a espécie B. tomentosa, os autores relataram DB de $581 \mathrm{~kg} \cdot \mathrm{m}^{-3}$ e DEpcs de 2,70 Gcal.m ${ }^{-3}$, ou seja, valores próximos daqueles obtidos neste estudo para T. vulgaris. A similaridade das características energéticas das madeiras do cerrado pode ser importante para subsidiar estudos futuros de manejo florestal. Além disso, as pesquisas devem se concentrar na identificação de espécies que apresentam maior ocorrência e que possuam propriedades semelhantes àquelas tradicionalmente utilizadas para fins energéticos. 


\subsection{Rendimentos da carbonização e qualidade do carvão vegetal}

Com relação ao rendimento gravimétrico em carvão vegetal (RGCV), o valor médio de 34,25\% encontrado nesse estudo foi maior que o apresentado em outros trabalhos que utilizaram a mesma metodologia para produção de carvão vegetal. Costa et al. (2014) ao avaliar o desempenho energético de cinco espécies do cerrado (Luehea divaricata, Casearia sylvestris, Guazuma ulmifolia, Rapanea ferruginea e Trema micranta) relataram valor médio para RGCV de 32,29\%. Enquanto que, Santos et al. (2016) e Protásio et al. (2021) encontraram valores médios variando de $31,25 \%$ a $35,80 \%$, respectivamente, para clones de Eucalyptus. Os rendimentos gravimétricos em carvão vegetal obtidos nesta pesquisa foram similares aos reportados na literatura para as carbonizações da madeira de Eucalyptus. De maneira geral, o rendimento gravimétrico em carvão vegetal é diretamente influenciado pelos teores de lignina e extrativos presentes na madeira (COSTA et al., 2014). Portanto, os resultados encontrados indicam que as madeiras do cerrado apresentam composição química apropriada para maximizar a produtividade de carvão vegetal em sistemas de carbonização tradicionalmente utilizados no Brasil. Os resultados de Marques et al. (2020), obtidos para madeiras do cerrado, corroboram com essa afirmação. Os autores reportaram teores de lignina insolúvel e de extrativos variando de 24,51$30,24 \%$ e de 5,99-13,18\%, respectivamente. Além disso, as espécies $A$. cearensis e $M$. urundeuva apresentaram madeiras mais densas e, consequentemente, isso pode resultar em melhor produtividade dos fornos de carbonização.

Quanto ao rendimento em carbono fixo, as espécies $M$. urundeuva e T. vulgaris se destacaram com $25,29 \%$ e $26,54 \%$, respectivamente. Esse rendimento indica que a cada tonelada de madeira de $M$. urundeuva carbonizada será obtido $\approx 253 \mathrm{~kg}$ carbono fixo. Por outro lado, a carbonização de uma tonelada de madeira de T. vulgaris resultaria em maior produtividade de carbono fixo $(265 \mathrm{~kg})$, ou seja, uma diferença de aproximadamente $4,7 \%$. No entanto, a densidade da madeira de T. vulgaris é considerada baixa (493 kg. $\left.\mathrm{m}^{-3}\right)$, enquanto $M$. urundeuva apresentou densidade média (706 kg.m ${ }^{-3}$ ). Diferenças de densidade da madeira poderão influenciar diretamente a produtividade de carbono fixo nos fornos de carbonização. Nesse sentido, Protásio et al. (2021) simularam o efeito da densidade básica da madeira de clones de Eucalyptus na produtividade de carbono fixo em sistemas de carbonização utilizados no Brasil. Os autores constataram que considerando os fornos retangulares com capacidade nominal de $283 \mathrm{~m}^{3}$, clones de Eucalyptus com DB $<500 \mathrm{~kg} . \mathrm{m}^{-}$ 3 não favoreceram o uso eficiente do volume do forno e; portanto, isso pode resultar na produção de carbono fixo até $10 \%$ menor comparativamente a madeiras com DB >550 $\mathrm{kg} . \mathrm{m}^{-3}$. Portanto, espera-se que a produtividade dos fornos de alvenaria seja consideravelmente menor se a madeira de $T$. vulgaris for utilizada como matéria-prima, uma vez que essa espécie apresentou densidade baixa (ver tópico 3.1).

Segundo Vale et al. (2010), as propriedades DB, DRA e $\mathrm{DE}$ são correlacionadas positivamente. Isso significa que quanto maior a densidade básica da madeira, mais denso será o carvão vegetal produzido e maior será a sua densidade energética. Essa tendência foi confirmada no presente estudo, em que a espécie de madeira mais densa ( $M$. urundeuva) apresentou carvão com maior DRA e DE. Além disso, os resultados permitem afirmar que o carvão vegetal de M. urundeuva apresentará melhor resistência mecânica
(COSTA et al., 2014; ABREU NETO et al., 2020) e melhor aproveitamento nos altos fornos siderúrgicos devido a maior massa de carvão disponível por unidade de volume (ARAÚJO et al., 2018). Os carvões com DRA acima de 250 $\mathrm{kg} . \mathrm{m}^{-3}$ são ideais para a finalidade siderúrgica, pois proporcionam maior capacidade térmica por unidade de volume (ASSIS et al., 2016). Contudo, conforme relatado anteriormente, aspectos de qualidade do carvão vegetal e produtividade dos fornos de carbonização devem ser considerados para a correta indicação de espécies para essa finalidade.

Vale mencionar que a maior densidade do carvão vegetal está diretamente relacionada com o estoque de carbono fixo e com o consumo específico de carvão vegetal para produção de ferro gusa. Nesse aspecto, os carvões produzidos com as madeiras das espécies $A$. cearensis e $M$. urundeuva apresentaram estoque de carbono fixo de $306 \mathrm{~kg} \cdot \mathrm{m}^{-3}$ e $340 \mathrm{~kg} \cdot \mathrm{m}^{-3}$, respectivamente. Esses valores foram consideravelmente superiores aos observados para o carvão vegetal de T. vulgaris $\left(227 \mathrm{~kg}\right.$ de carbono fixo por $\left.\mathrm{m}^{3}\right)$. Portanto, espera-se maior produtividade de ferro gusa com a utilização dos carvões produzidos a partir das madeiras de $A$. cearensis e $M$. urundeuva.

Para a utilização do carvão vegetal deve ser analisado o TCF, TMV e TCz, pois estas características possuem significativas correlações com o poder calorífico e com o desempenho do carvão nos altos fornos siderúrgicos (REIS et al., 2012; COSTA et al., 2014), sendo importante carvão com alto TCF $(>70 \%)$, TMV equilibrado $(20-25 \%)$ e baixos valores de $\mathrm{TCz}(<5 \%)$. Observa-se efeito significativo de espécie sob a composição química imediata do carvão vegetal. Notoriamente, as espécies estudadas apresentaram TCF e TCZ do carvão vegetal adequados, com destaque a $T$. vulgaris que apresentou melhores valores para ambas as propriedades $(\mathrm{TCF}=76,32 \%$; TCZ $=1,14 \%$ ), o que culminou no maior PCS do carvão vegetal $\left(7729 \mathrm{kcal}^{\mathrm{k}} \mathrm{kg}^{-1}\right)$. O equilíbrio entre TMV e TCF para produção do aço é importante, pois valores de TMV acima da faixa mencionada implicam na redução de TCF. Além disso, baixos teores de carbono fixo resultam em decréscimo da eficiência na redução do minério de ferro nos altos fornos siderúrgicos devido, principalmente, ao aumento do consumo específico de biorredutor para produção de uma tonelada de ferro-gusa (ASSIS et al., 2016).

Os resultados obtidos nesta pesquisa indicam que as espécies $A$. cearensis e $M$. urundeuva apresentam maior potencial para produção de carvão vegetal para fins siderúrgicos, enquanto o carvão vegetal de T. vulgaris é mais indicado para fins domésticos. Apesar da espécie T. vulgaris apresentar menor produtividade por forno de carbonização, a qualidade do carvão vegetal foi superior comparativamente às demais espécies mencionadas. Por exemplo, os teores de carbono fixo e de cinzas e poder calorífico do carvão vegetal de T. vulgaris foram melhores comparativamente aos carvões de $A$. cearensis e $M$. urundeuva. Finalmente, novas pesquisas deverão ser realizadas visando verificar com mais precisão os valores de variação da densidade da madeira de T. vulgaris e, consequentemente, sua aptidão para a produção de carvão vegetal em grande escala para fins de redução do minério de ferro e produção de ferro-gusa.

\section{CONCLUSÕES}

As espécies $M$. urundeuva, A. cearensis e T. vulgaris oriundas do cerrado goiano apresentaram madeira com potencial 
tecnológico adequado para geração direta de energia e produção de carvão vegetal. As madeiras das espécies $M$. urundeuva e $A$. cearensis apresentaram melhor desempenho energético devido aos maiores valores de densidade básica e densidade energética.

As propriedades da madeira (densidade básica, estoque de carbono, hidrogênio elementar e relação $\mathrm{H} / \mathrm{C}$ ) foram influenciadas apenas pelo fator espécie.

O teor de carbono fixo e o poder calorífico superior das madeiras do cerrado reduziram longitudinalmente. Tendência contrária foi observada para o teor de materiais voláteis.

Os carvões vegetais das espécies analisadas apresentaram potencial siderúrgico, especialmente o carvão da espécie $M$. urundeuva, que se destacou com os maiores valores de rendimento em carvão vegetal, densidade relativa aparente e densidade energética. $\mathrm{Na}$ ordem, as melhores espécies foram: M. urundeuva, A. cearensis e T. vulgaris.

Futuros estudos deverão ser conduzidos visando promover a domesticação das espécies $M$. urundeuva, $A$. cearensis e T. vulgaris. Além de estudos específicos de manejo florestal para viabilizar o uso energético sustentável das madeiras dessas espécies.

\section{AGRADECIMENTOS}

Os autores expressam seus sinceros agradecimentos a CAPES (código de financiamento 001) e ao CNPq (processo $\left.\mathrm{n}^{\circ} 306793 / 2019-9\right)$ pelo auxílio financeiro e ao Laboratório Multiusuário de Biomateriais da Universidade Federal de Lavras pelo apoio nos ensaios laboratoriais.

\section{REFERÊNCIAS}

ABREU NETO, R.; ASSIS, A. A.; BALLARIN, A. W.; HEIN, P. R. G. Effect of final temperature on charcoal stiffness and its correlation with wood density and hardness. SN Applied Sciences, v. 2, p. 1020, 2020. DOI: $10.1007 / \mathrm{s} 42452-020-2822-0$

ADAMOVICS, A.; PLATACE R.; GULBE, I.; IVANOVS, $\mathrm{S}$. The content of carbon and hydrogen in grass biomass and its influence on heating value. Engineering for Rural Development, v. 1, p. 1277-1281, 2018.

AMERICAN SOCIETY FOR TESTING MATERIALS. D1762-84: Standard Test Method for Chemical Analysis of Wood Charcoal. Philadelphia: ASTM International, 2007. 2p.

AMERICAN SOCIETY FOR TESTING MATERIALS ASTM. E711-87: Standard test method for gross calorific value of refuse-derived fuel by the bomb calorimeter. Philadelphia: ASTM International, 2004. 8p.

ARAÚJO, A. C. C.; COSTA, L. J.; BRAGA, P. P. C.; NETO, R. M. G.; ROCHA, M. F. V.; TRUGILHO, P. F. Propriedades energéticas da madeira e do carvão vegetal de Cenostigma macrophyllum: subsídios ao uso sustentável. Pesquisa Florestal Brasileira, v. 38, n. 1, p. 1-9, 2018. http:/ /dx.doi.org/10.4336/2018.pfb.38e201701546

ASSIS, M. R.; BRANCHERIAU, L.; NAPOLI, A.; TRUGILHO, P. F. Factors affecting the mechanics of carbonized wood: literature review. Wood Science and Technology, v. 50, p. 519-536, 2016. DOI: https://doi.org/10.1007/s00226-016-0812-6

ASSOCIAÇÃO BRASILEIRA DE NORMAS TÉCNICAS.
NBR 11941: madeira: determinação da densidade básica. Rio de Janeiro: 2003. 6p.

AZEVÊDO, T. K. B.; BRITO, H. R.; LIMA, F. S.; ALMEIDA, A. M. C.; TREVISAN, L. F. A.; PEREIRA, A. V.; OLIVEIRA, E.; MEDEIROS, J. X. Determinação da densidade básica da madeira de Piptadenia stipulacea (Benth) Ducke e Amburana cearensis. Agropecuária Técnica, v. 31, n. 1, 2010.

BRAND, M. A. Energia de biomassa florestal. Rio de Janeiro: Interciência, 2010. 131p.

CARNEIRO, A. C. O.; CASTRO, A. F. N. M.; CASTRO, R. V. O.; SANTOS, R. C.; FERREIRA, L. P.; DAMÁSIO, R. A. P.; VITAL, B. R. Potencial energético da madeira de Eucalyptus sp. Em função da idade e de diferentes materiais genéticos. Revista Árvore, Viçosa, v. 38, n. 2, p. 375-381, 2014. DOI: 10.1590/S010067622014000200019

CARVALHO, P. E. R. Espécies arbóreas brasileiras. EMBRAPA Informação Tecnológica, Brasília, p. 1039, 2003.

CHAVES, A. M. B.; VALE, A. T.; MELIDO, R. C. N.; ZOCH, V. P. Características energéticas da madeira e carvão vegetal de clones de Eucalyptus spp. Enciclopédia Biosfera, Goiânia, v. 9, n. 17, p. 533-542, 2013.

COSTA, A. C. S.; LEAL, C. S.; SANTOS, L. C.; CARVALHO, A. M. M.; OLIVEIRA, A. C.; PEREIRA, B. L. C. Propriedades da madeira de cerne e alburno de Eucalyptus camadulensis. Ciência da Madeira, Pelotas, v. 8, n.1, p. $10-20,2017$. DOI: 10.12953/21776830/rcm.v8n1p10-20

COSTA, T.G.; BIANCHI, M.L.; PROTÁSIO, T.P.; TRUGILHO, P. F.; PEREIRA, A.J.; Qualidade da madeira de cinco espécies de ocorrência no cerrado para produção de carvão vegetal. Cerne, Lavras, v. 20, n. 1, p. 37-45, 2014. DOI: 10.1590/S0104-77602014000100005

CSANÁDY, E.; MAGOSS, E.; TOLVAJ, L. Quality of Machined Wood Surfaces. New York City: Springer International Publishing, 2015. 257p. DOI: 10.1007/9783-319-22419-0

DIAS, A. C. C.; MARCHESAN, R.; ALMEIDA, V. C.; MONTEIRO, T. C.; MORAES, C. B. Relação entre a densidade básica e as retrações em madeira de teca. Ciência da Madeira, v. 9, n. 1, p. 37-44, 2018. DOI: 10.12953/2177-6830/rcm.v9n1p37-44

FERREIRA, D. F. SISVAR: a computer analysis system to fixed effects split plot type designs. Revista Brasileira de Biometria, v. 37, n. 4, p. 529-535, 2019. DOI: https://doi.org/10.28951/rbb.v37i4.450

FORTALEZA, A. P.; FILHO, J. J. P. N.; CERETTA, R. P. S.; BARROS, D. S.; SILVA, S. S. Biomassa de espécies florestais para produção de carvão vegetal. Ciência Florestal, Santa Maria, v. 29, n. 3, p. 1436-1451, 2019. DOI: $10.5902 / 1980509831639$

LIMA, E. A.; SILVA, H. D.; LAVORANTI, O. J.; Caracterização dendroenergética de árvores de Eucalyptus benthamii. Pesquisa Florestal Brasileira, Colombo, v. 31, n. 65, p. 9-17, 2011.

LIMA, M. D. R.; PATRÍCIO, E. P. S.; BARROS JUNIOR, U. O.; ASSIS, M. R.; XAVIER, C. N.; BUFALINO, L.; TRUGILHO, P. F.; HEIN, P. R. G.; PROTÁSIO, T. P. Logging wastes from sustainable forest management as alternative fuels for thermochemical conversion systems 
in Brazilian Amazon. Biomass and Bioenergy, v. 140, p. 105660, 2020 . DOI: https://doi.org/10.1016/j.biombioe.2020.105660

LOUREIRO, B.A.; VIEIRA, T.A.S.; COSTA, L.J.; SILVA, A.B.; ASSIS, M.R.; TRUGILHO, P.F. Selection of superior clones of Corymbia hybrids based on wood and charcoal properties. Maderas. Ciencia y tecnologia, v. 21, n. 4, p. 619-630, 2019.

MARQUES, R. D.; CUNHA, T. Q. G.; CHAGAS, M. P.; VENTUROLI, R. D.; BELINI, G. B.; YAMAJI, F. M.; SETTE JÚNIOR, C. R. Wood quality of five species of the Cerrado for energy purposes. Scientia Forestalis, v. 48, n. 125, e3225, 2020. DOI: https://doi.org/10.18671/scifor.v48n125.11

MMA_MINISTÉRIO DO MEIO AMBIENTE. Biomas, Bioma cerrado. 2020. Disponível em: < https://www.mma.gov.br/biomas/cerrado.html>. Acesso em: 25 set. 2020.

MORAES, M. D. A.; SANTOS, J. H.; LIMA, P. A. F.; CONEGLIAN, A.; SOUSA JÚNIOR, A. D.; CANTO, J. L.; SETTE JUNIOR, C. R. Bioenergia com resíduos do desdobro da madeira de Pinus caribaea var. hondurensis. Revista de Ciências Agrárias, Lisboa, v. 42, n. 2, p. 241 250, 2019. DOI: https://doi.org/10.19084/rca.17170

NEVES, T. A.; PROTÁSIO, T. P.; COUTO, A. M.; TRUGILHO, P. F.; SILVA, V. O.; VIEIRA, C. M. M. Avaliação de clones de Eucalyptus em diferentes locais visando à produção de carvão vegetal. Pesquisa Florestal Brasileira, Colombo, v. 31, n. 68, p. 319-330, 2011.

OLIVEIRA, A. C.; CARNEIRO, A. C. O.; VITAL, B. R.; ALMEIDA, W.; PEREIRA, B. L. C.; CARDOSO, M. T.; Parâmetros de qualidade da madeira e do carvão vegetal de Eucalyptus pellita F. Muell. Scientia Forestalis, Piracicaba, v. 38, n. 87, p. 431-439, 2010.

PROTÁSIO, T. P.; LIMA, M. D. R.; SCATOLINO, M. V.; SILVA, A. B.; FIGUEIREDO, I. C. R.; HEIN, P. G. R.; TRUGILHO, P. F. Charcoal productivity and quality parameters for reliable classification of Eucalyptus clones from Brazilian energy forests. Renewable Energy, v. 164, p. 34-45, 2021. DOI: https://doi.org/10.1016/j.renene.2020.09.057

PROTÁSIO, T. P.; SCATOLINO, M. V.; ARAÚJO, A. C. C.; OLIVEIRA, A. F. C. F.; FIGUEIREDO, I. C. R.; ASSIS, M. R.; TRUGILHO, P. F. Assessing proximate composition, extractive concentration, and lignin quality to determine appropriate parameters for selection of superior Eucalyptus firewood. BioEnergy Research, v. 12, p. 626-641, 2019. DOI: https://doi.org/10.1007/s12155-019-10004-x

PROTÁSIO, T. P.; TRUGILHO, P. F.; NEVES, T. A.; VIEIRA, C. M. M. Análise de correlação canônica entre características da madeira e do carvão vegetal de Eucalyptus. Scientia Forestalis, Piracicaba, v. 40, n. 95, p. 317-326, 2012.
PROTÁSIO, T. P.; BUFALINO, L.; TONOLI, G. H. D.; COUTO, A. M.; TRUGILHO, P. F.; GUIMARÃES JÚNIOR, M. Relação entre o poder calorífico superior e os componentes elementares e minerais de biomassa vegetal. Pesquisa Florestal Brasileira, Colombo, v. 31, n. 66, p. 113-122, 2011.

REIS, A. A.; PROTÁSIO, T. P.; MELO, I. C. N. A.; TRUGILHO, P. F.; CARNEIRO, A. C. O.; Composição da madeira e do carvão vegetal de Eucalyptus urophylla em diferentes locais de plantio. Pesquisa Florestal Brasileira, Colombo, v. 32, n. 71, p. 277-290, 2012.

SANTOS, R. C.; CARNEIRO, A. C. O.; VITAL, B. R.; CASTRO, R. V. O.; VIDAURRE, G. B.; TRUGILHO, P. F.; CASTRO, A. F. N. M. Influência das propriedades e da relação siringil/guaiacil da madeira de eucalipto na produção de carvão vegetal. Ciência Florestal, Santa Maria, v. 26, n. 2, p. 657-669, 2016. DOI: https://doi.org/10.5902/1980509822765

SANTOS, R. C.; CARNEIRO, A. C. O.; PIMENTA, A. S.; CASTRO, R. V. O.; MARINHO, I. V.; TRUGILHO, P. F.; ALVES, I. C. N.; CASTRO, A. F. N. M. Energy potential of species from forest management plan for the Rio Grande do Norte State. Ciência Florestal, Santa Maria, v. 23, p. 491-502, 2013. DOI: https://doi.org/10.5902/198050989293.

SANTOS, R. C.; CARNEIRO, A. C. O.; CASTRO, A. F. M.; CASTRO, R. V. O.; BIANCHE, J. J.; SOUZA, M. M.; CARDOSO, M. T. Correlation of quality parameters of wood and charcoal of clones of Eucalyptus. Scientia Forestalis, Piracicaba, v. 39, p. 221-230, 2011.

SILVA, D. A.; MULLER, B. V.; KUIASKI, E. C.; ELOY, E.; BEHLING, A.; COLAÇO, C. M. Propriedade da madeira de Eucalyptus benthamii para a produção de energia. Pesquisa Florestal Brasileira, Colombo, v. 35, n. 84 , p. $481-485,2015$.

SILVA, J. M. C.; SANTOS, M. P. D. A importância relativa dos processos biogeográficos na formação da avifauna do Cerrado e de outros biomas brasileiros. In: SCARIOT, A.; SOUSA FILHO, J. C.; FELFILI, J.M. (Ed.). Cerrado: ecologia, biodiversidade e conservação Ministério do Meio Ambiente, Brasília, 2005. p. 224-233.

SOARES, V. C.; BIANCHI, L. M.; TRUGIKHO, P. F.; PEREIRA, A. J.; HOFLER, J. Correlações entre as propriedades da madeira e do carvão vegetal de híbridos de eucalipto. Revista Árvore, Viçosa, v. 38, n. 3, p. 543549, 2014. DOI: 10.1590/S0100-67622014000300017

TERRA, T. D. R. Avaliação físico-química do carvão de cachamorra (Sclerolobium paniculatum vogel) e pau-terra de folha larga (Qualea grandiflora mart.). Bioenergia em Revista: Diálogos, v. 07, n. 2, p. 120 - 133, 2017.

VALE, A. T.; DIAS, I. S.; SANTANA, M. A. E. Relações entre as propriedades químicas, físicas e energéticas da madeira de cinco espécies do cerrado. Ciência Florestal, Santa Maria, v. 20, n. 1, p. 137-145, 2010. DOI: $10.5902 / 198050981767$ 